\title{
MODELOS DE BIOÉTICA
}

\section{Rafael Amo Usanos ${ }^{1}$}

Resumen: La bioética ya es una ciencia madura que admite el uso del metalenguaje para afrontar el reto del pluralismo. En este trabajo de metabioética se pretende, en primer lugar, comparar algunas de las más importantes propuestas de modelos bioéticos. En segundo lugar, mediante una metodología analítica, se expondrán los dos grandes grupos de resultados. Por una parte, los tres tipos de criterios utilizados para la construcción de modelos: antropológico, ético y biofilosófico. Por otra parte, se sacará a la luz las precomprensiones que subyacen a los discursos bioéticos que explican el pluralismo y dificultan el diálogo.

Palabras clave: modelos de bioética, metabioética, bioética

\section{Bioethical models}

Abstract: Bioethics is a mature science that allows for the use of metalanguage in order face the challenge of pluralism. In this work on metabioethics, the aims are, firstly, to compare some of the most important proposals of bioethic models. Secondly, by means of an analytical methodology two large groups of results will be presented: on the one hand, in terms of the three types of criteria used for the construction of models (anthropological, ethical and biophilosophical); and on the other hand, the presuppositions that underlie the bioethical discussions that explain pluralism and make the dialogue more difficult will be brought to light.

Keywords: bioethics models, metabioethics, bioethics

\section{Modelos de bioética}

Resumo: A bioética já é uma ciência madura, que admite o uso da metalinguagem para entrentar o desafio do pluralismo. Neste trabalho de metabioética pretende-se, em primeiro lugar, comparar algumas das propostas mais importantes de modelos bioéticos. Em segundo lugar, mediante uma metodologia analítica, expor os dois grandes grupos de resultados; de um lado, os três tipos de critérios utilizados para a construção de modelos - antropológico, ético e biofilosófico -; por outro, "se sacará" à luz das "precomprensiones" que subjacem aos discursos bioéticos que explicam o pluralismo e dificultam o diálogo.*

Palavras-chave: modelos de bioética, metabioética, bioética

\footnotetext{
${ }^{1}$ Universidad Pontificia Comillas, España

Correspondencia: rafael.amo@comillas.edu
} 


\section{Introducción}

El pluralismo en bioética plantea un doble reto: uno práctico, por ejemplo, en el día a día de los comités de bioética; y otro teórico, para dar razón de la causa última de este pluralismo. Ante este doble reto se adoptan varias posturas, desde aquel que - con cierto complejo de superioridadafirma que todos los demás están equivocados, a quien se limita a dejar constancia del pluralismo aprovechando su diversidad. En este trabajo se pretende bucear en el pluralismo bioético en busca de sus razones últimas.

Se parte de una convicción previa, que pone de manifiesto Gómez-Heras: la razón última del pluralismo ético es la opción por un determinado tipo ideal y por un valor tomado como referente. Tipo ideal es un concepto cercano a los de "modelo" o "paradigma", utilizados en teoría de la ciencia, "una categoría epistemológica con la que interpretamos un fenómeno histórico-social en sus peculiaridades individuales refiriéndolo a un valor cultural, religioso, ético, etc.”(1:29). Así se afirma que existen opciones previas al discurso bioético que predisponen la respuesta.

Para llevar a cabo este trabajo de metabioética, se propone utilizar un método comparativo-analítico, que se considera el más adecuado. En un primer momento, en el comparativo, mediante la revisión bibliográfica de las distintas taxonomías de modelos de bioética que se encuentran en la literatura, se podrá comparar los distintos tipos ideales utilizados y los valores referenciales. En el segundo momento, en el analítico, se pretende llegar al subsuelo de la razón última del pluralismo, al estudiar sus semejanzas y diferencias. En este subsuelo se espera encontrar las precomprensiones determinantes que justifican — no del todo racionalmente- el pluralismo bioético.

\section{Estudio sincrónico}

En la amplísima literatura bioética hay muy pocos estudios específicos dedicados a ofrecer una taxonomía de modelos bioéticos. Donde se suelen encontrar estos trabajos es en las introducciones que algunos hacen a la presentación general de la bioética. Además, este trabajo es preferido por los autores más mediterráneos, frente a los anglo- sajones que le dedican muy poco tiempo. Así las cosas, se recogen a continuación once taxonomías significativas, expuestas de forma sincrónica, que se consideran suficientes para el objetivo propuesto.

\subsection{Raanan Gillon, Philosophical Medical Ethics} (1985)

En el libro Philosophical Medical Ethics(2) Gillon dedica dos capítulos a proponer una taxonomía de modelos bioéticos. Se trata de una taxonomía muy primitiva, no en cuanto a estructura sino en cuanto a los autores estudiados en cada modelo. No hay que olvidar que solo está escrita seis años despues de la obra de Beauchamp y Childress, que inaugura uno de los modelos sobre los que se moverán, a favor o en contra, gran parte de las propuestas bioéticas.

Gillon, para elaborar su taxonomía, utiliza un criterio ético que le permite dividir los modelos en dos grandes grupos: la ética construida en atención a la norma y la ética construida en atención a las consecuencias. Ahora bien, la primera está definida solo por oposición a la segunda. Gillon afirma que hay dos grandes categorías de teoría moral. Uno afirma que las respuestas a las preguntas morales sobre qué acciones son correctas y cuáles incorrectas dependen finalmente de la naturaleza de las consecuencias de esas acciones o acciones propuestas. No es sorprendente que este grupo de teorías morales se llame "consecuencialista”, y sus miembros más conocidos e importantes sean aquellas teorías que se agrupan bajo el nombre de "utilitarismo". La segunda categoría de teorías morales son las llamadas teorías "deontológicas". Al menos algunas de las explicaciones de las obligaciones morales que ofrece este grupo de teorías no son reducibles a consideraciones de consecuencias.

De este modo, para Gillon, el grupo considerado deontologista es un cajón de sastre en el que caben las éticas religiosas y las no religiosas. Al referirse a las éticas religiosas las divide entre las que se pueden considerar moral de normas (como las que se rigen por los diez mandamientos) o las que utilizan el concepto de "ley natural". En el grupo de las éticas deontológicas no religiosas incluye la ética de Kant, la causística (ya que en el fondo, 


\begin{tabular}{|c|c|c|}
\hline \multicolumn{2}{|c|}{ Criterio ético: en atención a la norma o a la consecuencia } \\
\hline \multicolumn{2}{|c|}{ Deontológicos } & \\
\hline Religiosos & No-religiosos & \\
\hline Moral de reglas & Kant & Utilitarismo \\
Ley natural & Casuística & \\
& Principialismo & \\
\hline
\end{tabular}

afirma, se acaba utilizando un principio ético) y el incipiente principialismo, en el que considera la ética de la justicia de Rawls. Por lo que se refiere al utilitarismo lo define como aquella teoría moral basada en el principio de maximización del bienestar. Por el año de publicación, es fácilmente comprensible que en ninguno de los grupos cite a autores de bioética. La bibliografía era todavía muy escasa.

\subsection{Diego Gracia, Fundamentos de bioética (1989)}

Diego Gracia(3) ofrece una clasificación de los modelos bioéticos apoyada en los modelos éticos que, según su opinión, se han dado en la historia: el ontológico y el epistemológico.

Los del grupo ontológico tienen una estructura deductiva a partir de unos primeros principios. Los del grupo epistemológicos tienen su origen en la Crítica de la razón pura, de Kant, ya que, al poner su objetivo en analizar las condiciones de posibilidad de un conocimiento científico, empujó a la ética a utilizar una metodología no deductiva.

Sobre este esqueleto básico, Gracia ofrece una taxonomía cuádruple de los modelos bioéticos. Como pertenecientes al primer grupo considera el modelo ontológico o principialista, y el modelo deontológico o formalista. Como pertenecientes al segundo grupo cuenta el modelo epistemológico o decisionista y el modelo axiológico o del conflicto de valores.

El modelo ontológico o principialista, en el que considera fundamentalmente la bioética de la Iglesia católica, se fundamenta en que las cosas, en tanto que reales, son siempre buenas. Cierto que hay cosas malas, pero la maldad, ya sea física o moral, no la tienen las cosas en tanto que reales, sino solo en tanto que tales. Del ser real surgen los principios.

El modelo deontológico o formalista arranca de Kant. La moralidad no se puede fundamentar sobre principios heterónomos, aunque sean metafísicos, sino en un principio autónomo. Este es el carácter autolegislador del ser humano, que propone al hombre como fin en sí mismo, como principio de carácter formal.

Este modelo puede ser especificado como fuerte o débil. En su versión fuerte, representada por Apel y Habermas, es muy similar al ontológico. En su versión débil prima el procedimentalismo, muy cercano al convencionalismo. Los ejemplos más claros son John Rawls y Tristram Engelhardt.

Bajo el nombre de modelo epistemológico o deci-

\begin{tabular}{|c|c|c|c|c|}
\hline \multicolumn{3}{|c|}{ Criterio ético: en atención al método deductivo o no deductivo } \\
\hline Principialista & \multicolumn{2}{|c|}{ Formalista } & Decisionista & \multicolumn{2}{|c|}{ Axiológico } \\
\hline & Fuerte & Débil & $\begin{array}{c}\text { Metodología } \\
\text { de conflicto de } \\
\text { valores }\end{array}$ & $\begin{array}{c}\text { Ética de } \\
\text { situación }\end{array}$ \\
\hline Iglesia católica & $\begin{array}{c}\text { Apel- } \\
\text { Habermas }\end{array}$ & $\begin{array}{c}\text { Rawls- } \\
\text { Engelhadrt }\end{array}$ & $\begin{array}{c}\text { Pluralismo y } \\
\text { ética civil }\end{array}$ & $\begin{array}{c}\text { Buchanan, Nozick, Rickert, } \\
\text { Weber, Brentano, Scheler, } \\
\text { Hursell, Hartmann }\end{array}$ \\
\hline
\end{tabular}


sionista, Diego Gracia recoge las teorías del pluralismo y la ética civil. Las presenta como métodos de búsqueda de consenso ante la necesidad de que una sociedad proteja el bien común por medio de la llamada "moral civil". Estos métodos utilizan fundamentalmente el casuismo, tomado de otras ciencias como el derecho y la medicina.

El modelo axiológico o de conflicto de valores surge, según Diego Gracia, para superar la dicotomía entre el craso positivismo y el ontologismo ingenuo. En la búsqueda de un fundamento material a la moralidad se descubre la idea de "valor". En esta corriente incluye Gracia los nombres de David Ross Buchanan, Robert Nozick, H. Rickert y M. Weber, F. Brentano, M. Scheler, E. Husserl y N. Hartmann. Entre todos, Gracia distingue los que siguen la metodología del conflicto de valores y los que adoptan el método de la ética de situación.

\subsection{Elio Sgreccia, Manuale di bioetica (1991)}

Elio Sgreccia(4) utiliza un criterio combinado de antropología y ética según el cual propone como gran frontera la ley de Hume, que divide entre el cognitivismo y el no cognitivismo, y que contiene en sí los citados elementos antropológicos y éticos. En este sentido, los no cognitivistas piensan que los valores no pueden ser objeto de conocimiento y de afirmaciones calificables como verdaderas o falsas. Por el contrario, los cognitivistas buscan una fundamentación racional y objetiva de los valores y las normas morales.

De este modo, Sgreccia propone una taxonomía de siete modelos bioéticos:

El primero es el de la ética descriptiva y modelo sociobiológico. Afirma que, según esta perspectiva, la sociedad en su evolución produce y cambia valores y normas, que son funcionales para su desarrollo, igual que los seres vivos en su evolución biológica han desarrollado ciertos órganos con la finalidad de que cumplan una función y, en definitiva, para mejorar su propia existencia.

El segundo es el modelo subjetivista o liberal radical. Este cae del lado del no cognitivismo e incluye el neoiluminismo, el liberalismo ético, el existencialismo nihilista, el cientifismo neopositivista, el emotivismo y el decisionismo. La propuesta principal de todas estas corrientes es que la moral no se puede fundamentar ni en los hechos ni en los valores objetivos o transcendentes, sino solo en la opción autónoma del sujeto.

El tercero es el modelo pragmático-utilitarista, también en la corriente del no cognitivismo. El denominador común de estas diversas orientaciones de pensamiento es el rechazo de la metafísica y la desconfianza consiguiente respecto de la pretensión de poder alcanzar una verdad universal y, por tanto, una norma válida para todos en el plano moral. El principio básico es la relación costo/ beneficio.

El cuarto modelo que presenta es el contractualista. Lo describe como cercano al utilitarismo y basado también en el criterio del acuerdo intersubjetivo estipulado por la comunidad ética, es decir, por todos cuantos tienen la capacidad y la facultad de decidir.

El quinto modelo es el fenomenológico, especialmente el de Max Scheler y Nicolai Hartmann. Queda del lado del cognitivismo, ya que muestra una apertura a los valores éticos, una apertura definida como emocional e intuitiva a los valores; los valores éticos, sin embargo, están fundamentados a nivel emotivo y religioso. Debido a eso afirma que queda reducido a la subjetividad emocional

\begin{tabular}{|c|c|}
\hline \multicolumn{2}{|c|}{ Criterio ético y antropológico: la ley de Hume } \\
\hline Cognitivista & No-Cognitivista \\
\hline Fenomenológico & Ética descriptiva y modelo sociobiológico \\
Principialismo & Subjetivista o liberal radical \\
Personalismo & Pragmático-utilutarista \\
& Contractualista \\
\hline
\end{tabular}


y que, por esto mismo, no puede aspirar a tener validez universal. El horizonte sigue siendo un horizonte social, por lo demás difícil de formular.

El sexto modelo es el de la ética de los principios, el llamado principialismo, que se remite a Beauchamp y Childress. Dice de este modelo que afirma la necesidad de ciertos principios morales, pero cuya justificación sigue siendo imprecisa.

Por último, propone el modelo personalista, que considera el más adecuado para resolver las antinomias de los modelos precedentes y para fundamentar, al mismo tiempo, la objetividad de los valores y las normas.

\subsection{Carlo Cafarra, Introducción al Manual de bioética general (1994)}

Carlo Caffarra(5) utiliza un mapa de los modelos éticos para exponer la clasificación de los diversos modelos bioéticos. Partiendo de un estudio de la racionalidad práctica, Cafarra afirma que hay tres grandes tipos de respuestas a la naturaleza de esta racionalidad: la de D. Hume, la de E. Kant y la de santo Tomás.

Sobre la respuesta de D. Hume, afirma que la clave de comprensión está en su idea de "acto humano", para el cual la razón no tiene ninguna capacidad motiva y motivante: solo las pasiones empujan a actuar.

Desde el punto de vista de Caffarra, la postura de Kant se define por el hecho de que la razón práctica es pura cuando se determina a actuar sin ninguna condición empírica: cuando se determina según los principios absolutos a priori.

A la postura de santo Tomás dedica más tiempo, porque es la que considera verdadera. Después de sentar la definición de racionalidad práctica (el conocimiento del bien del hombre) y explicar los tres momentos del conocimiento práctico, está en disposición de afirmar que un acto es éticamente justificable cuando es un acto verdaderamente prudente, cuando el juicio de la conciencia es verdadero, esto es, iluminado y guiado por la ley moral.

Sobre este mapa de la ética clásica, Caffarra dibuja la cartografía de la moderna bioética. De la postura de Hume hace derivar todos los modelos utilitaristas. Bajo esta denominación designa la argumentación ética que se propone justificar una propuesta práctica sobre la base de sus consecuencias positivas y negativas, contrapuestas según un balance de bienes y males previstos. Por su parte, la racionalidad práctica, de ascendencia kantiana, se plasma en aquellos modelos bioéticos que ponen el centro en el principio de universalización: Singer, Hare y Habermas. Y, finalmente, el modelo argumentativo de tinte tomista va a dar lugar a todas las éticas de clara vocación antropológica, como son los personalismos.

\subsection{Dionigi Tettamanzi, Bioetica (1996)}

Este autor(6) propone con claridad un criterio antropológico para clasificar en dos grandes grupos a las corrientes bioéticas. Así, los dos grandes grupos son, por una parte, los modelos éticos inaceptables y, por otro, el modelo ético personalista.

El primer gran grupo está caracterizado por su concepción del hombre y su libertad. Este gran grupo da cabida a tres modelos. El primero es uno de tipo radical y nihilista, que se funda sobre la libertad concebida como valor único y absoluto. El segundo modelo ético de este grupo es el sociológico-utilitarista, que asume como criterio el valor de un determinado país o de un cierto tiempo o, sobre todo, el coste-beneficio. El tercer modelo es el científico tecnológico, cuyo punto fundamen-

\begin{tabular}{|c|c|c|}
\hline \multicolumn{2}{|c|}{ Criterio ético: modelos de estudio de la racionalidad práctica } \\
\hline Hume & Kant & Aristóteles-Santo Tomás \\
\hline Utilitarismo & Singer, Hare y Habermas & Personalismo \\
\hline
\end{tabular}

\begin{tabular}{|l|l|l|l|}
\hline \multicolumn{3}{|c|}{ Criterio antropológico: ¿qué es el hombre? } \\
\hline \multicolumn{3}{|c|}{ Inaceptables } & Personalismo \\
\hline Radical-Nihilista & Sociológico-utilitarista & Científico-tecnológico & Personalismo \\
\hline
\end{tabular}


tal consiste en afirmar la manipulabilidad total por parte del hombre. El segundo gran grupo es denominado "ético personalista", que para Tettamanzi es el único válido, porque la persona aparece en él como un valor objetivo, transcendente, intangible $y$, por tanto, normativo.

2.6. Jorge José Ferrer y Carlos Álvarez, Para fundamentar la bioética (2003)

Ferrer y Álvarez, en su libro común Para fundamentar la bioética(7), ofrecen una exposición de los modelos bioéticos. En esta obra se consideran dos grandes grupos de bioéticas: la angloamericana y la mediterránea, clasificación que viene a ser una simplificación de la taxonomía de naturaleza ética que ofrece Norman Daniels.

Daniels explica esta topografía, como él la denomina, en respuesta a la cuestión que considera clave: si la ética ha de estar dominada por los principios, quienes gobernarían en la ética, o, por el contrario, si hay que dar prioridad a los juicios éticos en su contexto. Así, el mapa ético se mueve entre dos polos: las teorías éticas y los juicios particulares.

En este mapa, de abajo arriba, los polos éticos tal y como los describen- son: 1.- Juicios particulares y acciones; 2.- Reglas; 3.- Principios; 4.- Teorías éticas. Así, con una imagen muy descriptiva, hablan de un Reino Medio con una serie de colinas fortificadas que son las fuerzas de los principialistas. Con vistas a dicho Reino Medio hay un terreno elevado más etéreo que está ocupado por las bandas errantes de habitantes del Reino Alto (Uplanders), que representan a los que dan valor a las teorías éticas. Abajo, en el valle, viven los Lowlanders, contextualistas y casuistas, y algunos otros que desconfían de las altas esferas.
Sobre esta descripción Ferrer y Álvarez construyen su distinción entre una bioética angloamericana y otra mediterránea. El criterio para esta clasificación no queda expuesto con claridad: la bioética mediterránea respondería a los lowlanders de Daniels, mientras que los uplanders y midlanders corresponderían a la bioética angloamericana.

De este modo, la bioética angloamericana incluye el principialismo, el paradigma casuístico, el paradigma de las virtudes, la bioética del permiso de H. T. Engelhardt, la ética médica comunitarista, la bioética del feminismo, la bioética utilitarista de Peter Singer, el pragmatismo clínico y el paradigma de la moralidad común.

Por su parte, en la bioética mediterránea quedan incluidas la bioética en Italia y la ética formal de bienes, y el principialismo jerarquizado de Diego Gracia. Dentro de la bioética en Italia estudia las bioéticas de inspiración católica, deteniéndose especialmente en la obra de Elio Sgreccia, y las de inspiración laica, estudiando con más atención a Scarpelli.

\subsection{Maurizio Chiodi, Modelli teorici in bioetica (2005)}

En uno de los escasos estudios monográficos sobre los modelos bioéticos(8), Maurizio Chiodi presenta su taxonomía de los modelos bioéticos. Propone una clasificación basada en las diversas corrientes éticas y su posición a favor o en contra del principialismo. Habla así de la bioética de los principios; de la tradición aristotélica; de la ética y bioética del cuidado, y, por último, de la fenomenología, hermenéutica y ética narrativa.

El primer grupo, al que denomina bioética de los principios, tiene su origen en la influencia anglófona. Esta categoría de bioética de los principios

Criterio ético: el papel de las teorías éticas y los juicios particulares

\begin{tabular}{|c|c|}
\hline Lowlander & Midlander \\
\hline Bioética mediterránea & Bioética angloamericana \\
\hline $\begin{array}{l}\text { Ética formal de bienes, } \\
\text { principialismo jerarquizado y } \\
\text { bioética en Italia (personalismo } \\
\text { de inspiración católica y bioética } \\
\text { de inspiración laica). }\end{array}$ & $\begin{array}{l}\text { Principialismo, el paradigma casuístico, el paradigma de las } \\
\text { virtudes, la bioética del permiso de H. T. Engelhardt, la ética médica } \\
\text { comunitarista, la bioética del feminismo, la bioética utilitarista de } \\
\text { Peter Singer, el pragmatismo clínico y el paradigma de la moralidad } \\
\text { común. }\end{array}$ \\
\hline
\end{tabular}


se caracteriza por aceptar, de forma más o menos crítica, los principios de Beauchamp y Childress. En este grupo se incluye la bioética de los principios de Beauchamp e Childress, la bioética contractualista — cuyo autor principal es Robert M. Veatch-; la bioética de la autonomía o del permiso, de Engelhardt; la ética utilitarista de Singer y la bioética laica en Italia, que incluye el utilitarismo de M. Mori y la ética utilitarista y analítica de Lecaldano.

El segundo grupo, el de la categoría de bioética de tradición aristotélico-tomista, agrupa a los autores que reaccionan contra la bioética de los principios. El mismo Chiodi reconoce la heterogeneidad de este grupo, puesto que no existe una reflexión unívoca. Incluye así, dentro de este modelo, la filosofía de la medicina de Edmund Pellegrino, la bioética de la virtud de Hauerwas y, también, la nueva casuística de Toulmin y Jonsen.

En tercer lugar, Chiodi examina el modelo de la bioética del cuidado. También considera a esta corriente en la línea contraria al principialismo. Su característica fundamental es su orientación feminista, aunque también existe otra desarrollada en otros contextos. Entre las autoras del primer grupo distingue a Joan Baker Miller, Nancy Chodorow, Carol Gilligan, Nel Noddings, Virginia Held y Luisella Battaglia. Por lo que se refiere a la bioética del cuidado en otros contextos, Chiodi cita a Daniel Callahan y Warren Th. Reich.

En cuarto y último lugar, examina Chiodi la bioética del ámbito de la fenomenología, la hermenéutica y la ética narrativa. De este modelo afirma que es una "alternativa alla bioetica di derivazione contrattualistica analitica e utilitaristica”. Dentro de este grupo advierte dos corrientes. Por un lado, la que representan Richard M. Zaner, R. Charon, H. Brody, S. Kay Toombs y Diego Gracia y, por otro, se refiere al personalismo fenomenológico de Paolo Cattorini.

\subsection{James F. Childress, The Oxford Handbook of Bioethics (2007)}

En el fondo, para Childress(9) solo hay dos modelos de bioética, la basada en principios y la basada en casos. En el modelo basado en principios distingue entre principialismos consecuencialistas, principialismos deontológicos y acercamientos principialistas.

En los principialismos consecuencialistas, a su vez, distingue entre dos tipos: los métodos de las consecuencias de los actos (Joseph Fletcher o Peter Singer, y el del informe Warnok) y los métodos de las consecuencias de las normas (Brad Hooker), según se apoyen en los efectos de determinados actos o en los efectos del tipo de acción.

En los principalismos deontológicos incluye a la bioética de inspiración kantiana, que contiene expresiones como "dignidad humana", "autonomía" y "respeto por la persona". Ahora bien, siguiendo a Onora O'Neill, distingue entre los puramente kantianos y los de inspiración religiosa, es decir, los que hablan de respeto a la autonomía o los que hablan de santidad de la vida. Como ejemplo de los primeros habla de Robert M. Veatch y Kevin Wildes; como ejemplo de los segundos cita a Engelhardt, Juan Pablo II y Paul Ramsey.

En el acercamiento principialista incluye fundamentalmente su propia obra colectiva con Beau-

\begin{tabular}{|c|c|c|c|c|c|}
\hline \multicolumn{6}{|c|}{ Criterio ético: a favor o en contra del principialismo } \\
\hline \multirow{2}{*}{$\begin{array}{l}\text { Ética de los } \\
\text { principios }\end{array}$} & \multicolumn{5}{|c|}{ Postura en contra del principialismo } \\
\hline & $\begin{array}{l}\text { Tradición } \\
\text { aristotélica }\end{array}$ & Ética de & cuidado & Fen & enología \\
\hline $\begin{array}{c}\text { Principialismo, } \\
\text { contractualismo, } \\
\text { bioética del } \\
\text { permiso, } \\
\text { utilitarismo y } \\
\text { bioética laica } \\
\text { italiana }\end{array}$ & $\begin{array}{l}\text { Bioética de } \\
\text { Pellegrino, } \\
\text { bioética de la } \\
\text { virtud y nueva } \\
\text { casuística }\end{array}$ & Feminista & $\begin{array}{c}\text { No- } \\
\text { Feminista }\end{array}$ & $\begin{array}{c}\text { Solo } \\
\text { descriptivos }\end{array}$ & $\begin{array}{l}\text { Personalismo } \\
\text { fenomenológico }\end{array}$ \\
\hline
\end{tabular}




\begin{tabular}{|c|c|c|c|c|c|}
\hline \multicolumn{6}{|c|}{ Criterio ético: el método de la ética } \\
\hline \multicolumn{5}{|c|}{ Método deductivo } & $\begin{array}{l}\text { Método } \\
\text { inductivo }\end{array}$ \\
\hline Principialismo c & nsecuencialistas & $\begin{array}{r}\text { Princi } \\
\text { deont }\end{array}$ & $\begin{array}{l}\text { alismos } \\
\text { lógicos }\end{array}$ & $\begin{array}{l}\text { Acercamientos } \\
\text { principialistas }\end{array}$ & \multirow[b]{2}{*}{ Casuística } \\
\hline $\begin{array}{c}\text { Consecuencialismo } \\
\text { de actos }\end{array}$ & $\begin{array}{c}\text { Consecuencialismo } \\
\text { de normas }\end{array}$ & Kantianos & $\begin{array}{l}\text { De } \\
\text { inspiración } \\
\text { religiosa }\end{array}$ & $\begin{array}{c}\text { Virtudes } \\
\text { Cuidado } \\
\text { Comunitarismo }\end{array}$ & \\
\hline
\end{tabular}

champ y la ética de virtudes (Rosalind Hurtshouse y a E. Pellegrino), del cuidado (Carol Gilligan como la fundadora y Rita Manning y Annette Baier) o el comunitarismo (Ezekiel Emanuel y Raanan Gillon), ya que, a su modo de ver, tanto la virtud, como el cuidado o el bien común pueden jugar el papel de principios.

Los métodos basados en casos son aquellos que utilizan una metodología inductiva "bottom-up" para la justificación ética. Para exponerlo se apoya en la obra de Albert Jonsen y Stephen Toulmin.

\subsection{Fernando Pascual, Modelli di bioética (2007)}

Pascual(10) parte de una primera distinción, la que hay entre bioética religiosa y laica. Se centra en esta segunda para cuya taxonomía, que recoge tres modelos, utiliza un criterio a medio camino entre la ética y la antropología: la relación entre el individuo y otras instancias más generales (sociedad, especie humana, diversas formas de vida, medioambiente), lo que le lleva a la relación entre bien general y bien individual.

Así, habla de unas bioéticas sociobiológicas, para las cuales el ser humano no es el centro, y en ellas incluye a Peter Singer, y las bioéticas mediambientales, entre las que cita la de Potter y Aiken.

El contrapunto a las citadas bioéticas sociobiológicas son las bioéticas individualistas y subjetivistas, que hunden sus raíces en lugares tan dispares como el existencialismo de Sartre, el pensamiento libertario de Marcuse, el emotivismo moral del Hume y el liberalismo de Popper, Nozick y Dworkin. De este modo, los dos grandes representantes de este modelo son, por un lado, Engelhardt y, por otro, el movimiento de bioética laica de Uberto Scarpelli.

A medio camino entre ambos, Pascual sitúa el utilitarismo.

\subsection{Antonio Tarantino, Natura dell'uomo e mo-} delli di bioetica (2016)

Para Tarantino(11) todo comienza con la fractura en el paradigma de unidad racional y la aparición de otro nuevo paradigma: el de la racionalidad plural, en el que se renuncia a la verdad y se construye una ética sin ella.

Esta ruptura es lo que hace posible, para Taranti-

\begin{tabular}{|c|c|c|c|}
\hline \multicolumn{2}{|c|}{ Bioética laica } & \multirow{2}{*}{ Bioética religiosa } \\
\hline \multicolumn{2}{|c|}{ Criterio antropológico y ético: bien individual-bien general } & \\
\cline { 1 - 2 } Primacía del bien general & $\begin{array}{c}\text { Equilibrio bien general/ } \\
\text { bien individual }\end{array}$ & $\begin{array}{c}\text { Primacía del bien } \\
\text { individual }\end{array}$ & \\
\hline Bioéticas sociobiológicas & Utilitarismo & $\begin{array}{c}\text { Bioéticas individualistas } \\
\text { y subjetivistas }\end{array}$ & \\
\hline $\begin{array}{c}\text { Bioéticas espiecistas y } \\
\text { medioambientales }\end{array}$ & $\begin{array}{c}\text { Existencialismos, } \\
\text { pensamiento libertario, } \\
\text { emotivismo moral y } \\
\text { liberalismo }\end{array}$ & \\
\hline
\end{tabular}


no, la existencia de pluralidad en el campo bioético. En concreto, en esta ciencia se manejan tres tipos básicos de concepto de "naturaleza humana", lo que va a dar lugar a tres grandes grupos de modelos.

El primero de ellos es el compuesto por los modelos liberal-radical y pragmático-utilitarista, y agrupa a aquellos autores que hacen de su concepto de libertad como autodeterminación el elemento definitorio de la naturaleza humana.

Así las cosas, en este grupo enumera la bioética de los principios; el necontratcualismo y la ética procedimental; el liberalismo de Rawls; el utlilitarismo, en el que considera a Singer, Rachels y Engelhardt; el subjetivismo decisionista de Scarpelli y dos variantes de este modelo que son las de Lecaldano y Mori.

El segundo grupo de autores está formado por aquellos para los que el hombre no es pensado como un átomo sino unido a la especie humana con la que comparte la misma sustancia. Dentro de este segundo grupo, al que denomina "modelos de racionalidad y universalidad de la acción y de la racionalidad práctica abierta a la transcendencia", considera la bioética de Habermas (bioética del discurso entre libertad y deber), la de Hans Jonas (bioética de la responsabilidad) y la de Dworkin (bioética laica de la sacraliadad de la vida, la religión sin Dios).

El tercer y último grupo es el que se constituye en torno a un concepto de naturaleza que no se deja llevar por los mandatos de la antropología poscartesiana, aquella que no acepta un concepto de naturaleza preconstituida, que no sostiene que el individuo sea el constructor de su propia entidad a través de un proceso de ontodetermianción, que no acepta la cognoscibilidad de normas éticas absolutas y que no presenta el concepto de naturaleza como un dato opaco privado de significado intrínseco. Es decir, no privilegia el hacer sobre el ser, sino que privilegia el ser y el hacer armónicamente unidos. En este modelo considera la bioética católica y la del personalismo ontológicamente fundado de Elio Sgreccia.

\subsection{Rafael Amo, Vida y ética (2017)}

La propuesta de esta obra(12) supone una gran novedad respecto de los demás estudios sobre modelos de bioética. Propone que el concepto "vida", estudiado por la filosofía de la biología, es el que permite ofrecer una taxonomía coherente de modelos de bioética.

En un amplísimo e inédito estudio de filosofía de la biología sobre el concepto "vida", pone de manifiesto que esta ciencia maneja tres conceptos de vida asociados a tres momentos de la historia de la cosmología.

La vida, dicho inicialmente y de forma general, es la actividad del ser vivo. Pero, a lo largo de la historia, esta actividad se ha explicado de tres formas diferentes, que se corresponden con las tres grandes cosmovisiones, comprensiones de la naturaleza e imágenes del universo que se han dado en el pensamiento.

El primer periodo, el paradigma clásico, está dominado por la cosmología del Timeo de Platón, diálogo en el que el filósofo entiende la vida como la actividad del alma. El segundo periodo, el pa-

\begin{tabular}{|c|c|c|}
\hline \multicolumn{3}{|c|}{ Criterio antropológico: concepto de naturaleza humana } \\
\hline Naturaleza y libertad & Naturaleza social & Naturaleza preconstituida \\
\hline $\begin{array}{c}\text { Modelos liberal radical y } \\
\text { pragmático-utilitarista }\end{array}$ & $\begin{array}{c}\text { Modelos de racionalidad y } \\
\text { universalidad de la acción, y de } \\
\text { la racionalidad práctica abierta } \\
\text { a la transcendencia }\end{array}$ & \\
\cline { 1 - 2 } $\begin{array}{c}\text { Bioética de los principios; el } \\
\text { necontratcualismo y la ética } \\
\text { procedimental; el liberalismo } \\
\text { de Rawls; el utlilitarismo, y el } \\
\text { subjetivismo decisionista }\end{array}$ & $\begin{array}{c}\text { Bioética del discurso, bioética católica y personalismo } \\
\text { de la responsabilidad y bioética } \\
\text { laica de la sacralidad de la vida }\end{array}$ & \\
\hline
\end{tabular}


radigma mecanicista, queda dominado por la cosmología del mecanicismo, en la que domina lo no vivo y en la que el concepto de vida se duplica, apareciendo la vida de la res cogitans y la vida de la res extensa, explicada la primera en términos psicológicos y la segunda en términos mecánicos.

En el tercer periodo - la cosmología del paradigma sistémico- los sistemas abiertos son la pieza clave que hay que resolver para comprender el resto del puzle del universo, mientras que la vida es la actividad de unos determinados sistemas abiertos por mantenerse lejos del equilibrio.

Esta es la clave de esta propuesta de taxonomía: el concepto de vida puede ser el puente entre las dos ciencias (la filosofía de la biología y la bioética). Así utiliza el concepto de tipo ideal: estos tres conceptos de vida se pueden utilizar como tipo ideal para la construcción de una taxonomía de los modelos bioéticos.

Este criterio, procedente de la filosofía de la biología, sirve para proponer tres grandes modelos de bioética. En primer lugar, la bioética del paradigma clásico, que se caracteriza por utilizar el concepto vida de la cosmología del Timeo. En este grupo considera a la bioética personalista -Elio Sgreccia y Roberto Andorno- y de la virtud Pellegrino-.

En segundo lugar, el modelo de la bioética del paradigma mecanicista, cuya característica es utilizar un concepto vida proveniente del mecanicismo, considera la bioética animalista - Singer, Franccione y Regan-, el principialismo - Beauchamp y Childress_-, el principialismo europeo y Engelhartd-, Habermas y algunos autores de neuroética —-Gazzaniga y Glannon-.

Y, en tercer lugar, la bioética del paradigma sistémico, que recoge el concepto vida de la cosmología denominada sistémica. En este modelo considera a la bioética de inspiración zubiriana, a la bioética de la complejidad —Edgar Morin y su escuela - y a la bioética medioambiental _ Schweitzer, Leopold y Capra-.

\section{Análisis diacrónico}

Una vez expuestas estas taxonomías de modelos llega el momento del análisis que realizará en dos niveles.

\subsection{Primer nivel de análisis}

En un primer nivel, de simple observación, se puede comprobar que aparecen tres grandes grupos de criterios de clasificación: los éticos, los antropológicos y los de la filosofía de la naturaleza. Para la mayoría de los autores que intentan poner orden en el proceloso bosque de la bioética, el criterio más evidente es el ético: dependiendo del modelo de ética, así es el modelo de bioética. Para otros autores, mayoritariamente procedentes del ámbito católico, el criterio de clasificación es el antropológico, seguramente por la pretensión de éstos de sacar a la luz determinadas precomprensiones que hacen incompatible las respuestas bioéticas con la fe que profesan. Por último, el más reciente de los modelos bioéticos analizados utiliza un criterio procedente de la filosofía de la naturaleza, en concreto de la biología. Con él pretende ir más allá de los otros dos criterios, ya que considera que no dan razón en totalidad de las divergencias, especialmente porque suelen dejar fuera de su consideración a las bioéticas ambientales, y por la dificultad que los otros dos criterios manifiestan a la hora de clasificar el animalismo y el utilitarismo, lo que pone de manifiesto que su punto de partida no es suficiente.

\subsection{Segundo nivel de análisis}

El segundo nivel pretende sacar a la luz las precomprensiones determinantes (el tipo ideal), sobre

\begin{tabular}{|c|c|c|}
\hline \multicolumn{3}{|c|}{ Criterio biofilosófico: concepto de vida } \\
\hline Vitalismo & Mecanicismo & Sistémico \\
\hline $\begin{array}{l}\text { Personalismo } \\
\text { Bioética de la virtud }\end{array}$ & $\begin{array}{c}\text { Movimiento animalista, } \\
\text { la bioética principialista, } \\
\text { Habermas o autores de } \\
\text { neuroética, como Gazzaniga y } \\
\text { Glannon }\end{array}$ & $\begin{array}{c}\text { Bioética de inspiración } \\
\text { zubiriana, bioética del } \\
\text { pensamiento complejo } \\
\text { (Edgar Morin) y bioética } \\
\text { medioambiental }\end{array}$ \\
\hline
\end{tabular}




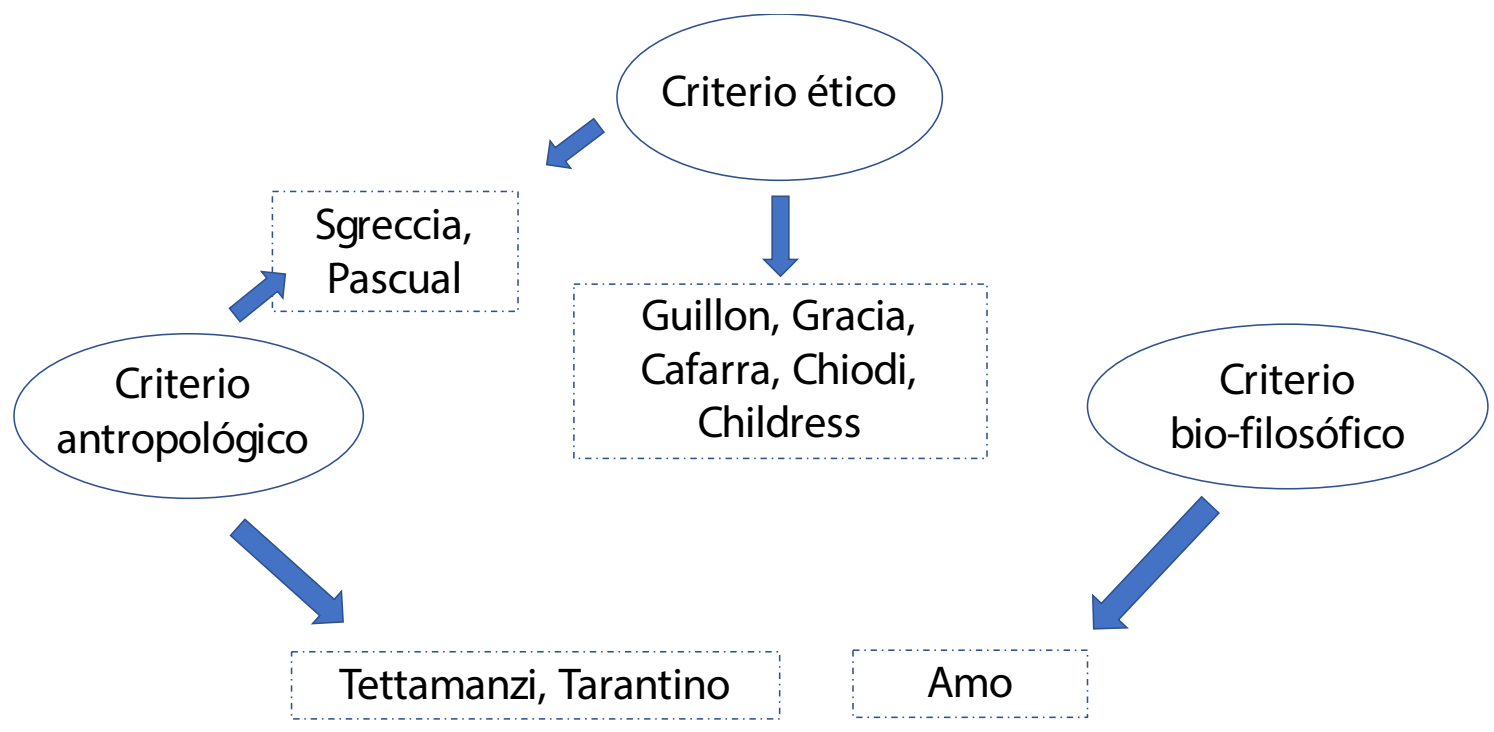

las cuales los autores construyen su bioética y que esta comparación de taxonomías de modelos de bioética ha permitido descubrir.

\section{a. La precomprensión de la objetividad normativa}

En la mayoría de las taxonomías expuestas hay un grupo reservado a la bioética de los principios que son afirmados de forma absoluta, es decir, sin justificar. En este sentido, son muy similares a las bioéticas de la virtud y las del cuidado. Estos elementos (principios, virtud, cuidado, etc.) son propuestos como valor referencial o tipo ideal sin discusión previa, a modo de precomprensión.

Muchos autores parten de la convicción de la existencia de una normatividad ética, ya sea transcendente (en el sentido de su origen en un ser superior) o inmanente (en el sentido de que la normatividad procede del mismo ser). Esta normatividad ética, se encuentre donde se encuentre (en la transcendencia o en la inmanencia), y se desarrolle como se desarrolle (en el concepto de naturaleza, de persona, dignidad, de autonomía $\mathrm{u}$ otros principios, etc.), es el horizonte de unas bioéticas que afirman, ya sea material o formalmente (con expresión kantiana), que existe la objetividad moral y esta afirmación es determinante.

b. La precomprensión del primado de la sensación

En casi todas las taxonomías hay una categoría especial reservada para el utilitarismo $-\mathrm{y}$ su pariente cercano, el animalismo- a la que por su importancia consideran como un modelo especial difícilmente reductible a otros. Esta precomprensión hunde sus raíces en la filosofía de la baja Edad Media, en concreto del encuentro entre el pensamiento oriental y el pensamiento de los llamados "pueblos germánicos", y que después se extendió con éxito por el pensamiento inglés de la escuela de York. Comienza cuando se pierde la categoría de símbolo como portadora de verdad, ya que solo lo sensible es real y, por tanto, verdadero. Este principio da el salto de la epistemología a la ética: si la verdad se percibe por los sentidos, lo que nos convierte en seres que conocen son estos mismos sentidos que compartimos animales y hombres. Esto iguala, epistemológicamente hablando, a hombres y animales, ¿por qué no éticamente? Los dos podemos sentir, los dos podemos sufrir, pues los dos deberemos tener la misma consideración ética. Este salto a la ética alcanza al concepto de bien y al principio utilitarista: el bien se mide en términos de bienestar sensible y lo útil en aquello que consiga la maximización del bienestar sensible.

c. La precomprensión del centro axiológico de lo real

No todas las taxonomías estudiadas dejan espacio para la bioética medioambiental, la de inspiración potteriana. A medio camino entre la metafísica, la cosmología y la antropología, aparece otra pre- 
comprensión que determina qué elemento de la realidad ocupa la cúspide de la jerarquía axiológica y, por tanto, es el mayor valor a proteger, al que se supeditan todos los demás. Para la mayoría de los modelos bioéticos es la persona, en sus múltiples definiciones, o el sujeto sentiente. Pero las taxonomías que recogen la bioética de inspiración potteriana apuntan a que el centro axiológico es la vida, que está por encima de los individuos que la viven. La vida es el valor referencial independientemente de quien la viva.

\section{Conclusión}

La bioética es una ciencia que ya ha alcanzado su madurez. Los trabajos de metabioética ya arrojan sus frutos. En este caso, el análisis comparativo de las diversas propuestas de modelos bioéticos permite llegar a las precomprensiones, no siempre conscientes, que dificultan el diálogo bioético. El trabajo realizado es un trabajo abierto en un doble sentido. En primer lugar, porque permite seguir recorriendo la literatura en busca de taxonomías propuestas por otros autores. En segundo lugar, porque se puede investigar el papel concreto de cada precomprensión en los debates particulares de cada asunto de bioética para ayudar al diálogo sincero libre de posiciones predeterminadas.

\section{Referencias}

1. García Gómez-Heras JM. Teorías de la moralidad. Introducción a la ética comparada. Madrid: Síntesis; 2004.

2. Gillon R. Philosophical Medical Ethics. Chichester-New York: John Wiley \& Sons; 1985.

3. Gracia D. Fundamentos de bioética. Madrid: EUDEMA; 1989.

4. Sgreccia E. Manuale di bioetica. Milano: Vita e Pensiero; 1991.

5. Cafarra C. Introducción general. En: Polaino-Lorente A, editor. Manual de bioética general. Madrid: Rialp; 1994: 23-30.

6. Tettamanzi D. Bioetica: Storia, problemi e riposte. Segrate: Piemme; 1996.

7. Ferrer JJ, Álvarez C. Para fundamentar la bioética. Madrid-Bilbao: Comillas-DDB; 2003.

8. Chiodi M. Modelli teorici in bioetica. Milano: FrancoAngeli; 2005.

9. Childress JF. Methods in Bioethics. In: Steinbock B, editor. The Oxford Handbook of Bioethics. New York: Oxford University Press; 2007.

10. Pascual F. Modelli di bioetica. Roma: Edizioni ART; 2007.

11. Tarantino A. Natura dell'uomo e modelli di bioetica. Milano: Guiffrè Editori; 2016.

12. Amo Usanos R. Vida y ética. Madrid: Síntesis; 2017.

Recibido: 21 de junio de 2018

Aceptado: 12 de julio de 2018 